\title{
Detailed Electromechanical Model of Ventricular Wedge
}

\author{
Alexander Kursanov ${ }^{1,2}$, Vladimir Zverev ${ }^{2}$, Leonid Katsnelson ${ }^{1,2}$, Olga Solovyova ${ }^{1,2}$ \\ ${ }^{1}$ Institute of Immunology and Physiology, Ural Branch of the Russian Academy of Sciences, \\ Ekaterinburg, Russia \\ ${ }^{2}$ Ural Federal University, Ekaterinburg, Russia
}

\begin{abstract}
We developed a three-dimensional computational model for describing electro-mechanical behavior of wedgeshaped preparation extracted from the left ventricular wall including excitation wave propagation, high-resolution geometry and fiber orientation. The cardiac tissue is simulated by an incompressible hyperplastic material. We used non-linear partial differential equations describing the deformation of the cardiac tissue, and a detailed "Ekaterinburg-Oxford" (EO) cellular model of the electrical and mechanical activity of the cardiomyocytes in the tissue. Electro-mechanical coupling in the model accounts for mechano-electric feedbacks both in the cells and in the tissue.

Numerical experiments with the model of the wedge preparation formed of initially identical cardiomyocytes revealed that electrical and mechanical interaction between the cells, as well as intracellular mechanoelectric feedbacks caused pronounced nonuniformity of their behavior.
\end{abstract}

\section{Introduction}

There is a wide evidence on the influence of mechanical conditions and mechanical activity on the electrical activity in myocardium, which is referred as the cardiac mechano-electric feedback (MEF) [1]. Mechanical effects on excitation have been registered in isolated cells, multicellular preparations and in the whole organ from various species [2]. However, the most of the experimental data shed a light on the MEF effects on micro(intracellular) and meso- (cellular) levels. Much less is known on how the MEF mechanisms are integrated and reveal themselves on macrolevels in the tissue and organ, where electrical and mechanical waves developing in myocardium interfere and influence each other within the entire multicellular sample. Integrative results of all these electro-mechanical processes have been observed in experiments on the whole heart. Taggart and Sutton did not find dispersion of repolarization in the intact ventricle despite significant transmural electrical nonuniformity of the cardimyocetes and suggested that interaction between cardiomyocytes in the tissue does contribute to the transmural alignment of the AP (action potential) duration [1]. On the other hand, there was shown that abnormalities in the pacing of the left ventricle may induce ventricular electrical remodeling [3] resulting in growth of the dispersion in the AP duration (APD) significantly. In particular, these authors showed that this electrical remodeling strongly correlates with the regional deformation pattern preconditioned by the excitation sequence. They also suggested that cardiac MEF might play a key role in cardiac memory [3]. That would be hardly possible to assess separate mechanisms revealing themselves in the MEF on the level of the whole heart. Nowadays, mathematical modeling seems the only possible way to uncover the interplay of all $\mathrm{MEF}$ mechanisms on the tissue level [4,5].

Experimental approach involving the left ventricular wedge preparations has been developed to study transmural electrophysiology and mechanical function in the multicellular segment of the ventricle [6]. Wedge preparations more recently were used in optical mapping studies [7]. Thus, isolated wedge preparation has become an important experimental tool in cardiac physiology.

In this study, we present a detailed electromechanical model including both intracellular and intercellular MEF mechanisms to study excitation-contraction coupling in the left ventricle wedge preparation.

\section{Mathematical model}

As the size of the cardiomyocytes is sufficiently small compared to the heart characteristic dimensions, any cardiac cell can be considered as an isopotential point of myocardial tissue. In this case, a wedge-shaped sample may be considered as a continuous medium where each cell (point) dynamically changes its position during the contractile cycle. On the other hand, the same cell has its own internal local geometry that also changes during the cycle, and just these local deformations of the cells produce together the movement of every one of them within the tissue - i.e. the total tissue deformation on the macrolevel. 
Thus, an electrical wave of excitation propagates along the dynamically deformable medium. Therefore, two geometrical spaces are considered in the model:

1. microspace representing cellular geometry;

2. macrospace representing the wedge geometry.

Both micro- and macro-circuits of the electromechanical and mechano-electric coupling in cardiac tissue are taken into account in the model (Figure 1). At the cellular level electromechanical coupling (EMC) between the membrane action potential (AP) generation and cellular contraction is mediated by the calcium activation of the cross-bridge attachment, while the mechano-electric feedback (MEF) is provided by the mechano-dependence of the calcium kinetics [8]. Cooperative influence of the attached cross-bridges on the kinetics of calcium-troponin complexes underlies this mechano-dependence [8]. At the tissue level, electrical waves of depolarization and repolarization and mechanical wave of deformation arising due to the electrical and mechanical coupling between cardiomyocytes also affect each other.

The model accounts for the electrical and mechanical anisotropy of the myocardial tissue associated with the orientation of the muscle fibers.

The Wedge preparation in the model is presented as a tissue sample in the form of a parallelepiped with a specified fibers orientation. The boundary conditions (see below) correspond to the experimental protocol [9].

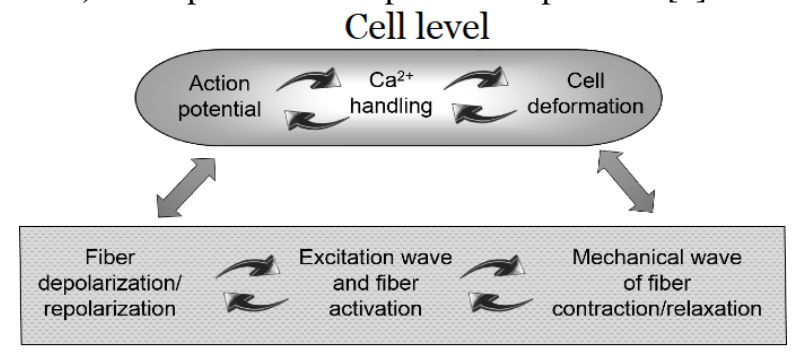

Tissue level

Figure 1. Scheme of electro-mechanical coupling in the myocardium at the cellular level and tissue level.

Electrical and mechanical activity of cells is described by the "Ekaterinburg-Oxford" mathematical model (EO model) of a single cardiomyocyte [10].

The electrical excitation of the myocardial tissue is defined by the reaction-diffusion equation. The first term of the equation determines the excitation spread through the tissue (macrolevel), and the second term describes the change in the membrane potential in the cells (points) due to the local transmembrane ionic currents (microlevel):

$$
\frac{\partial V}{\partial t}=d i v_{x} \cdot(D \nabla V)-\frac{1}{C_{m}} \cdot \sum i_{i o n}
$$

where $V$ is membrane potential of the cell, $C_{m}$ - membrane capacitance, $\sum i_{i o n}(x, t)$ - sum of the ion currents across the cell membrane, div - divergence operator in the current coordinates, $\nabla$ - the gradient operator, and $D$ - electrodiffusion coefficient matrix determining the diffusion velocities along and across the fiber orientation in the tissue.

Mechanical structure of individual cardiomyocytes within the tissue is represented by the rheological scheme shown in Figure 2.

The most of the myocardium passive mechanical properties we describe in the model on the macro-level. To do this, when incorporating the cellular model into the tissue model, we used the fact that the diagonal elements of the matrix $E$ (the strain gradient - see below) characterize the relative change in body length along the coordinate axes.

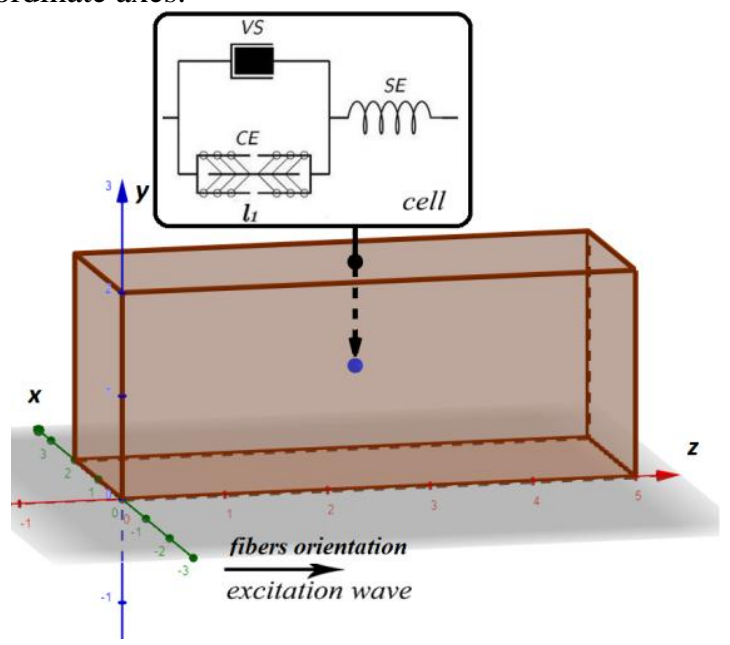

Figure 2. The scheme of wedge preparation. The rheological scheme shows mechanical structure of single virtual cardiomyocyte corresponding to a cell from the wedge, where the contractile element $(C E)$ is connected inseries to the passive elastic element $(S E)$ and in-parallel to the viscous element $(V S) . l_{l}$ is deformation of $C E$ relative to its slack length.

The key feature of the mechanical block of the model is relationship between global $(E)$ and local deformations of tissue, so that the local one along the direction of the muscle fiber is identified with the relative deformation of the cell $(l)$ :

$$
l=E m,
$$

where $m=F a_{0}\left(F a_{0}\right)^{T}, a_{0}$ is the direction of the muscle fiber before deformation, $F a_{0}$ indicates on the direction of the fiber after deformation.

The combined model of electromechanical activity of the myocardium is as follows:

$$
\begin{aligned}
& \frac{\partial V}{\partial t}=\operatorname{div}\left(J^{-1} q\right)-\frac{1}{C_{m}} \cdot \sum i_{i o n}(x, y, z, t), \\
& q=D \nabla V, D=d_{\perp}+\left(d_{\|}-d_{\perp}\right) m, \\
& \operatorname{div}\left(J^{-1} \tau\right)=0, \\
& \tau=\tau_{\text {act }}+\tau_{\text {pass }},
\end{aligned}
$$




$$
\begin{aligned}
\tau_{\text {act }} & =\sigma \cdot m, \\
\tau_{\text {pass }} & =\frac{C}{2}\left(e^{q}-1\right), q=\sum_{i=1}^{3} \sum_{k=1}^{3}\left(A_{i k} E_{i k}^{F}\right) E_{i k}^{F} .
\end{aligned}
$$

Equations (1') - (2) are written in Lagrange coordinates as compared to the term $\operatorname{div}(D \nabla V)$ in equation (1). This makes it possible to circumvent computational difficulties caused by the moving of the boundaries, and to use numerical methods for fixed domains. On the other hand, the complexity increases due to the calculating of the Jacobian $J$ and its reciprocal magnitude. Equation (3) is equilibrium equation for the continuous medium, based on Newton's third law, written using the Kirchhoff tensor. Also (3) assumes that the magnitude of the mass forces is small in comparison with the internal stress. Relations (6) reproduce passive properties of myocardial tissue.

The total stress is composed of active and a passive stresses (equation 4). Equation (5) serves to approximate the active stress, the magnitude of which is directed only along the fiber.

\section{Results}

In this work, we considered simulations of the electromechanical activity of a left ventricle wedge excised from the equatorial region of the LV wall. The transmural size of the LV wedge was based on data reported in [9]. To simplify the simulations both Laplacian and conductivity tensor in the equations (2) were assumed transversely isotropic. The fiber direction was assumed to be parallel to the axis $\mathrm{z}$, whereas the transmural direction was aligned with the axis $\mathrm{x}$ (see Fig. 2).

Figure 3 shows the typical results of calculations for the wedge myocardial sample in the form of a parallelepiped with initial sizes of $2 \mathrm{~mm}$ along axes $\mathrm{x}$ and $\mathrm{y}$ and $5 \mathrm{~mm}$ along axis $\mathrm{z}$ (see Figure 3 for $\mathrm{t}=0$ ).

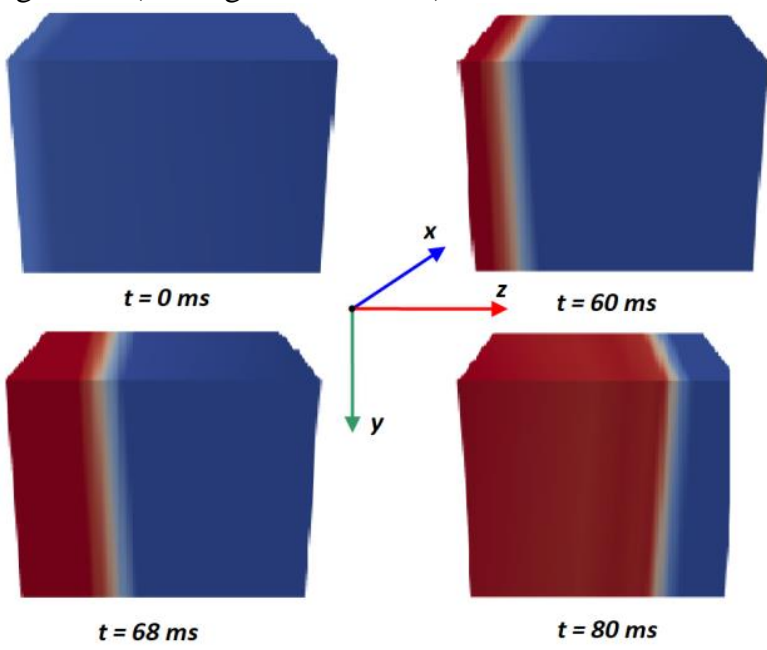

Figure 3. Spatial distribution of the transmembrane potential $V$ in the electromechanical simulation.
The body was divided into tetragonal elements by the following procedure: first, it was divided into 20 smaller parallelepipeds, and each of them was then decomposed into tetrahedrals.

The outer surface of the wedge was assumed electrically isolated (electrical boundary condition).

Since cardiac tissue contracts along the fiber direction, we observed an expansion of the wedge in the transmural direction due to its incompressibility.

The isotonic mode of the wedge contraction was simulated by the following mechanical boundary conditions imposed on the left and right sides of the sample (see Figures 2 and 3 ): the left one could not move along the axis $\mathrm{z}$, and an external load (preload) was applied to the opposite side. The cells on the left side of the wedge were electrically activated (initial condition). As a result, the AP propagated along the fiber direction forming flat front of the electrical wave. Figure 3 demonstrates the action potentials and local deformations in several cells disposed along the axis $\mathrm{z}$ (see Figure 3 ).
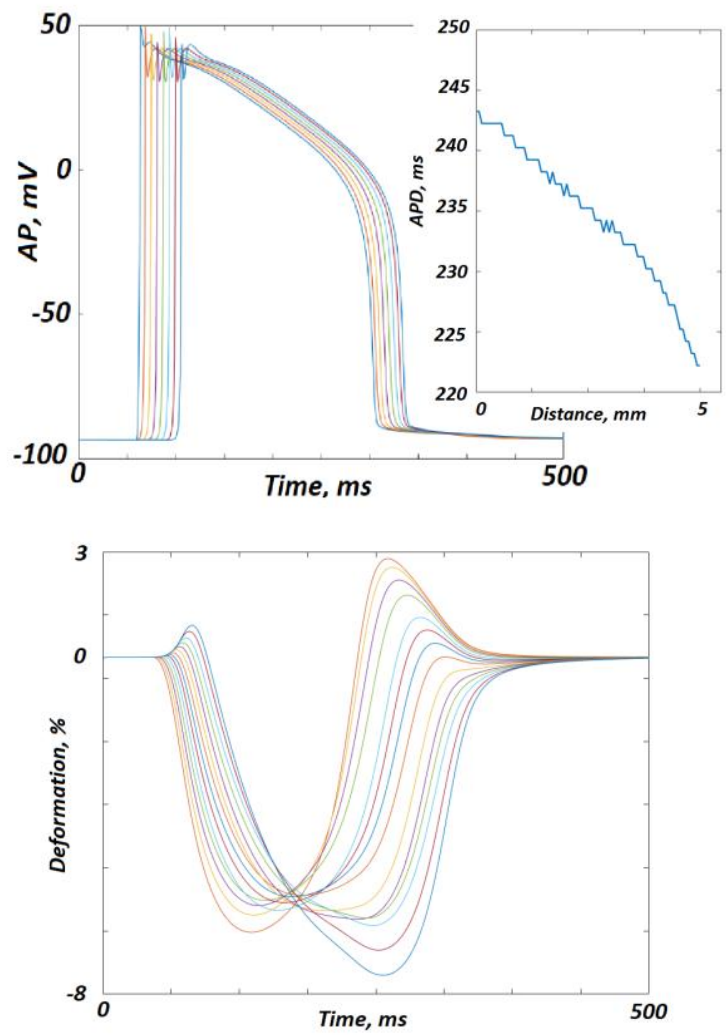

Figure 4. Top panel: Action potentials and their duration (APD) for various cells along the axis z. Bottom panel: deformations for various cells along the axis $\mathrm{z}$ in the electromechanical simulation (the cells were selected with a step of $0.2 \mathrm{~mm}$ from a middle fiber of the wedge).

Both panels of the Figure 4 show heterogeneous electromechanical behavior of the cells in the process of 
their mechanical and electrical interactions: along the wave the APD decreases and the pattern of cellular deformations changes. Both asynchronism of the cell excitation produced by the electric wave propagation and mechanoelectrical feedbacks (intracellular and intercellular ones) caused this functional heterogeneity even in a homogeneous sample formed from the identical virtual cardiomyocytes. Position of the cardiomyocytes in the wedge relative to the electrical wave front determined their mechanical and electrical behavior.

\section{Conclusion}

While both intracellular EMC and MEF have been widely discussed [11], the influence of mechanical interactions between the cells on the electrical wave propagation in the heart remains largely unappreciated. In this work, we presented an electromechanical model of the left ventricle wedge preparation suitable for analyzing the effects of cardiac tissue deformation on its electrophysiological pattern. The model makes it possible to investigate the effects of electric and mechanical interaction of cardiomyocytes on their function during contractions.

Using the electromechanical model of left ventricle wedge preparation of myocardium we have demonstrated that electrical and mechanical activity of cardiomyocytes strongly depends on their location within cardiac tissue and on the timing of their electrical activation during the contraction-relaxation cycles. Both factors seem to be important ones for the spatio-temporal organization of myocardium.

The influence of the local dynamic deformations on the AP generation in coupled cells and repolarization wave spread resulted from the intracellular MEF mechanisms, including cooperative mechanisms of $\mathrm{Ca}^{2+}$ activation of the cross-bridge attachment.

We revealed the role of mechano-electric interactions between the cells in producing AP duration gradient in the virtual preparation that helps to compensate the time delay in excitation between distant myocardial segments and to reduce the dispersion of repolarization within the strand as shown in the intact heart [1].

In the future, we plan to simulate the wedge preparations with different fiber orientations and initial stimulation regions and take into account transmural nonuniformity of cardiomyocytes inherent to the LV wall.

\section{Acknowledgements}

This work was carried out within the framework of the IIF UrB RAS themes (Nos. AAAA-A18-1180205900318) and was supported by RFBR (18-31-00416), the Program of the Presidium RAS \#27 (project AAAA-A18-
118020590030-1) and Act 211 Government of the Russian Federation, contract № 02.A03.21.0006.

\section{References}

[1] Taggart P, Sutton PM Cardiac mechano-electric feedback in man: clinical relevance. Prog Biophys Mol Biol 1999; 71:139-54

[2] Kamkin, A. G., Kiseleva, I. S. Mechanosensitivity in cells and tissues. Academia Publishing House Limited 2005: 1465

[3] Jeyaraj D., Wilson L.D., Zhong J., Flask C., Saffitz J.E., Deschenes I., Yu X., Rosenbaum D.S. Mechanoelectrical feedback as novel mechanism of cardiac electrical remodeling. Circulation. 2007;115: 3145-55.

[4] Trayanova, N. A., \& Rice, J. J. Cardiac electromechanical models: from cell to organ. Frontiers in Physiology 2011; 2 (43)

[5] Keldermann RH, Nash MP, Gelderblom H, Wang VY, Panfilov AV Electromechanical wavebreak in a model of the human left ventricle. Am J Physiol Heart Circ Physiol 2010; 299: H134-43

[6] Zhu, T. G., Patel, C., Martin, S., Quan, X., Wu, Y., Burke, J. F., ... \& Yan, G. X. Ventricular transmural repolarization sequence: its relationship with ventricular relaxation and role in ventricular diastolic function. European Heart Journal 2009; 30(3): 372-80

[7] Di Diego, J. M., Sicouri, S., Myles, R. C., Burton, F. L., Smith, G. L., \& Antzelevitch, C. Optical and electrical recordings from isolated coronary-perfused ventricular wedge preparations. Journal of Molecular and Cellular Cardiology 2013; 54: 53-64.

[8] Solovyova, O., Vikulova, N., Katsnelson, L. B., Markhasin, V. S., Noble, P. J., Garny, A., ... \& Noble, D. Mechanical interaction of heterogeneous cardiac muscle segments in silico: effects on $\mathrm{Ca}^{2+}$ handling and action potential. International Journal of Bifurcation and Chaos 2003; 13(12): 3757-82.

[9] Kawel, N., Turkbey, E. B., Carr, J. J., Eng, J., Gomes, A. S., Hundley, W. G., ... \& Lima, J. A. Normal left ventricular myocardial thickness for middle-aged and older subjects with steady-state free precession cardiac magnetic resonance: the multi-ethnic study of atherosclerosis. Circulation: Cardiovascular Imaging 2012; 5(4): 500-8.

[10] Sulman T, Katsnelson LB, Solovyova O, Markhasin VS Mathematical modeling of mechanically modulated rhythm disturbances in homogeneous and heterogeneous myocardium with attenuated activity of $\mathrm{Na}+\mathrm{K}+\mathrm{Pump}$. Bull Math Biol 2008; 70: 910-49.

[11] Kohl, P., Sachs, F., \& Franz, M. R. Cardiac mechano-electric coupling and arrhythmias. Oxford University Press 2011.

Address for correspondence.

Name. Alexander Kursanov

Full postal address. IIF UrB RAS, 620049 Pervomayskaya 106, Yekaterinburg, Russia

E-mail address. alexander.kursanov@gmail.com 